\title{
The use of point of care ultrasound (POCUS) in UAE emergency departments (ED)
}

\author{
Varsha Arjandas, Fatema Salem Al Ameri, Mehmood Chaudhry and Rasha Buhumaid* \\ Shaikh Khalifa Medical City, Abu Dhabi, United Arab Emirates.
}

Accepted 26 January, 2018

\begin{abstract}
Point of care ultrasound (POCUS) performed at bedsides is an invaluable tool and is used to evaluate clinical manifestations, to facilitate accurate diagnosis, and to assist procedures in emergency and critical care. This study aimed to characterize the current practice of emergency physician-performed POCUS in UAE Emergency Departments. An online survey of all government emergency departments in the UAE was conducted from January 2016 to August 2016 in order to determine how POCUS was used. A 20-question survey, including Emergency department demographics, ownership of ultrasound (US) equipment, and frequency of POCUS performance in general and by examination type, credentialing patterns, quality assurance methods, and billing patterns was conducted. Overall, $70 \%$ of respondents reported lack of training as the greatest barrier for the use of POCUS. Other shortcomings included not owning equipment, concerns regarding liability, not beneficial financially, and adequate coverage by other specialists. In conclusion, the study identified an underutilization of POCUS in UAE government Emergency Departments, with only a quarter of them performing POCUS regularly. Most of the respondents stated that attending a training course is the minimal requirement for the physicians to perform POCUS. Recent educational and training advancements might help to overcome this barrier.
\end{abstract}

Keywords: Ultrasound, point of care ultrasound (POCUS), emergency department.

${ }^{*}$ Corresponding author. E-mail: rbuhumaid@seha.ae.

\section{INTRODUCTION}

Point of care of ultrasonography (POCUS) is a convenient and prominently-growing imaging technique in the field of emergency medicine (Whitson and Mayo, 2016). POCUS is a safe and cost-efficient diagnostic modality that has been increasingly used in the Emergency Department (ED) (Choi et al., 2017; Atkinson et al., 2015).During the past 20 years many Emergency Physicians (EP) used focused ultrasound at bedsides in order to overcome challenging problems and at the same time providing care to critically ill patients (Chen et al., 2016).

POCUS was initially introduced in trauma as the Focused Assessment with Sonography (FAST) exam; however, with time this technique has been increasingly used in numerous other applications due to its movability and easy accessibility (Kameda and Taniguchi, 2016). With the advancement of ultrasound technology and increased need to take care of more patients quickly and cost-effectively by the EPs, there is a surge of POCUS, since it can reduce the cost of ultrasound, decrease the need for additional diagnostic testing, and increase the patient throughput and patient's satisfaction (Sanders et al., 2015). Additionally, POCUS is useful for the following functional clinical categories, such as accurate diagnoses, acute resuscitation, therapeutics or physiological monitoring, and as an aid to guide procedure in emergency and critical care (Atkinson et al., 2015). As a result, POCUS image acquisition and interpretation has become essential for Emergency Medicine (EM) residency training in majority of the academic EDs (Sanders et al., 2015). The significance of POCUS lies in its ability to specify a differential diagnosis based on clinical information from the patients records and immediate physical examination and also to take 
quick decision for further treatment (Choi et al., 2017). Studies have indicated that POCUS utilization has facilitated timely diagnosis and treatment intervention of unstable patients (Bashir et al., 2016). Hence, most emergency medical training programs worldwide have included POCUS in their curriculum. Despite its advantages, POCUS still remains underutilized in emergency situations. Several barriers are considered to be the reasons for underutilization of POCUS. Some of the probable barriers are lack of adequate skills and experience of the operators, challenges related to equipment and technology, obtainability of documentation template, unavailability of electronic storage to archive image, and absence of appropriate policies and procedures for quality assurance and billing (Choi et al., 2017).

The emergency medicine in the UAE has come a long way in a short time (Fares et al., 2014). Large, well equipped EDs are usually located in government hospitals in UAE, most of the EDs are staffed with medically or surgically trained physicians, with boardcertified EMs serving as consultants overseeing care.

The aim of this study is to characterize the current practice and attitudes of EPs performing POCUS in government EDs in UAE and also to explore their level of training and interest in developing POCUS skill and to determine the specific barriers to utilization of POCUS.

\section{METHODS}

An online questionnaire survey (Appendix) was conducted over a period of 8-months (January to August, 2016) in all EDs of government hospitals in the UAE to determine how POCUS was utilized in the EDs. Contact information of all UAE ED directors was obtained from scientific society directory. Urgent care centers and EDs of private hospitals were excluded. This study was approved by the Institutional Review Board (Ethics committee clearance no. REC-12.01.2016 [RS-412]). A 20-question online survey was designed using questions from previously published POCUS surveys' (Moore et al., 2006; Herbst et al., 2015). The questions addressed ED demographics, ownership of ultrasound (US) equipment, frequency of POCUS performance in general and by examination type, credentialing patterns, quality assurance methods, and billing patterns. The survey was deployed on a Google form. A link to the survey was sent via email to the directors of EDs. The survey was voluntary. No confidential information was requested, no risk of endangerment to patients, physicians, or others was conceivable, and no patient information was being solicited in this survey. The number of respondents was tracked via Google form and a reminder email was sent to non-respondents at every 4 weeks. After 5 months, telephone calls were made to several ED directors who had not yet responded. Survey responses were recorded onto Excel data sheets (Microsoft Corporation) via export from Google form. The survey data were summarized by means of chart and tables using Microsoft excel.

\section{RESULTS}

Twenty one EDs met the inclusion criteria and questionnaire was sent to them. Of these, ED directors from 12 hospitals completed the survey during the 8 month study period yielding a response rate of $57.1 \%$. Summary of the ED department characteristics are shown in Table 1. Among the responding EDs, $16.6 \%$ ( $\mathrm{n}$ $=2$ ) were smaller-volume EDs with annual visit volume of less than $40,000,41.6 \%(n=5)$ were medium-volume EDs with annual visit volume of 41,000 to 80,000 , and $41.6 \%(n=5)$ were large-volume EDs with annual visit volume of $>80,000$. $58 \%$ (7 out of 12 ) of the EDs reported that they have to limit ordering ultrasound studies from the radiology department and $16.6 \%$ (2 out of 12) EDs reported that radiology performed ultrasound is not available after regular working hours.

Overall, $58 \%$ (7 out of 12) of EDs reported owning at least one US machine. The mean number of ED-owned US machines was 2.57 (standard deviation 1.13).

$75 \%$ (9 out of 12) EDs reported using POCUS. 25\% (3 of 12) EDs reported using POCUS regularly for many applications, all of these EDs were large volume EDs. All of the EDs that reported never using POCUS were medium volume EDs. Figure 1 depicts the frequency of POCUS use by examination type. About $50 \%$ of the respondents mentioned that they regularly use POCUS in FAST examination, $42 \%$ mentioned they use POCUS regularly for aorta examination and basic echocardiography. However, for soft tissue/musculoskeletal, deep venous thrombosis, transabdominal pelvic, renal and biliary examinations, only 17 to $25 \%$ reported about regular use of POCUS and nearly 33 to $50 \%$ respondents reported of never using POCUS for these examinations (Figure 1).

The response from ED directors about reasons for not implementing any POCUS program is outlined in Figure 2. $70 \%$ (8 of 12) EDs reported lack of EP training to be the reason for not performing POCUS, $40 \%$ (5 of 12) said the technique is not financially beneficial, $40 \%$ (5 of 12) reported not owning US equipment, $40 \%$ (5 of 12) reported a concern regarding liability, and $30 \%$ of the respondents mentioned that they could not use POCUS due to adequate coverage by other specialists. According to $10 \%$ (1 of 10 ), the technique consumed too much time, $10 \%$ (1 of 12) reported the cost of the equipment to be too high, and $10 \%$ ( 1 of 12 ) mentioned about some form of resistance from radiology.

A total of $83 \%$ (10 of 12) UAE EDs reported regarding hospital ultrasonography privileges. $58 \%$ (7 of 12) reported "application-specific" hospital privileges (meaning EPs are granted privileges for specific ultrasound examinations) and 25\% (3 of 12) mentioned about "global" privileges (meaning EPs are granted the privilege for all emergency ultrasound examinations). $75 \%$ (9 of 12) EDs reported that attending a training course was the minimal required training for physicians to perform POCUS. Only $42 \%$ (5 of 12 ) reported to have a POCUS quality assurance process.

Lack of training $(70 \%)$, was detected to be the most common reason cited by ED directors for not implementing POCUS program (Figure 2). With regard to 
Table 1. Characteristics of EDs as reported by ED directors.

\begin{tabular}{lcc}
\hline ED patient visit per year & Number & Percentage (\%) \\
\hline 40,000 or less & 2 & 16.67 \\
$41,000-80,000$ & 5 & 41.67 \\
More than 80,000 & 5 & 41.67 \\
Number of full time physicians & & \\
Less than 5 & 1 & 8.33 \\
$5-10$ & 3 & 25.00 \\
$11-15$ & 2 & 16.67 \\
$16-20$ & 1 & 8.33 \\
More than 25 & 5 & 41.67 \\
& & \\
Percentage of staff Emergency Medicine trained & & \\
0 & 3 & 25.00 \\
$1-25 \%$ & 5 & 41.67 \\
$26-50 \%$ & 3 & 25.00 \\
$100 \%$ & 1 & 8.33 \\
\hline
\end{tabular}

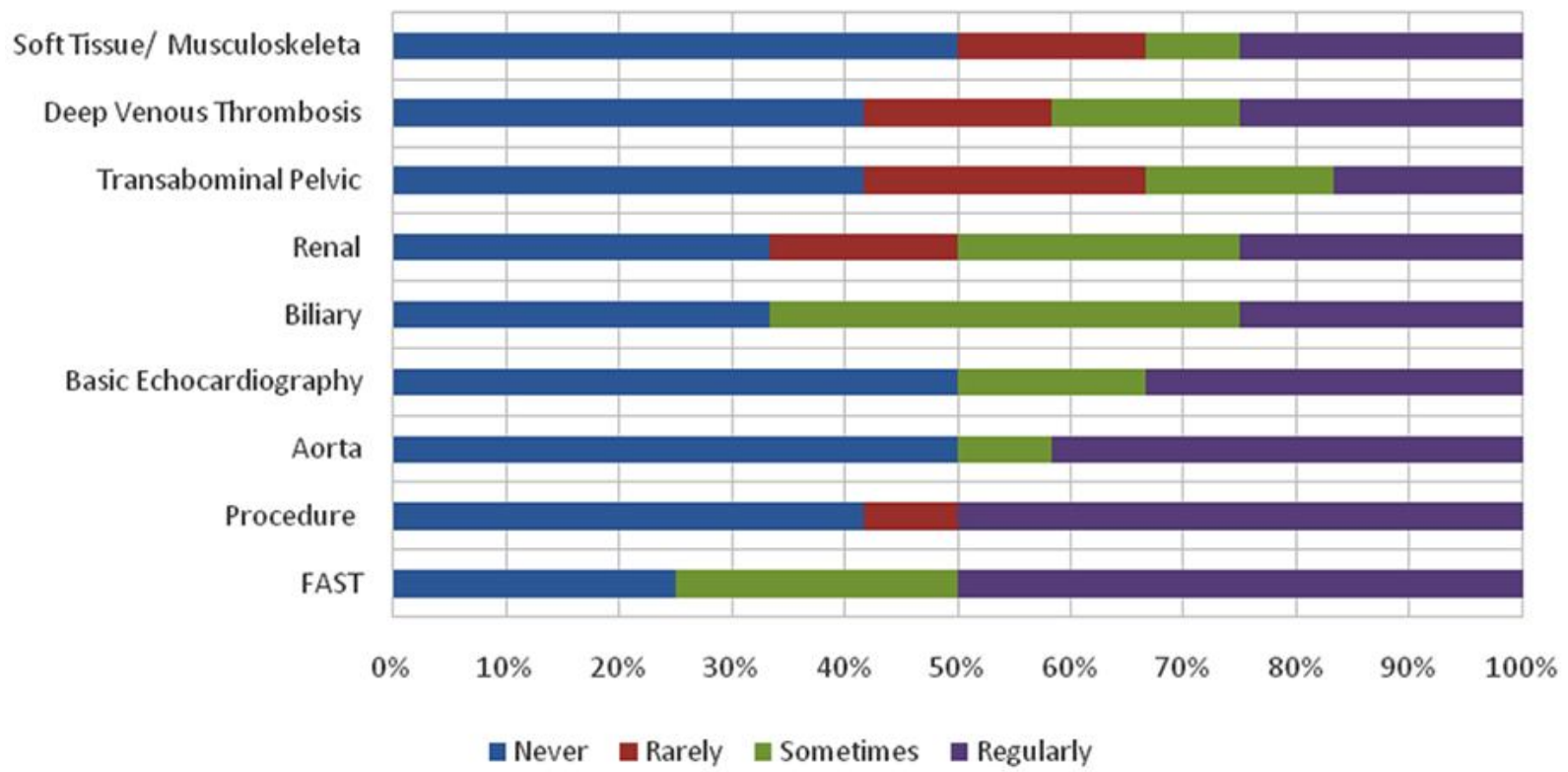

Figure 1. Frequency of POCUS use by examination type.

billing, only one (8.3\%) ED reported about billing separately for POCUS examination.

\section{DISCUSSION}

A significant enhancement in the use of POCUS in EDs across the world has been observed in the last decade. The policies and guidelines for the training and use of 'bedside ultrasound' have been devised by several credible organizations such as the ACEP, the American Institute of Ultrasound in Medicine (AIUM), American
Medical Association (AMA), and International Federation of Emergency Medicine (IFEM) (Bashir et al., 2016). In fact, the Accreditation Council for Graduate Medical Education (AGME) has made it compulsory to obtain ultrasonography training before the assessment of trainees in EM. However, it has been noticed that there is a slow acceptance of ultrasound by ED physicians outside USA.

Focused assessment with sonography in trauma (FAST) and extended FAST (eFAST) are widely available and may be performed quickly in real time. Recently, several research studies have inferred that bedside 


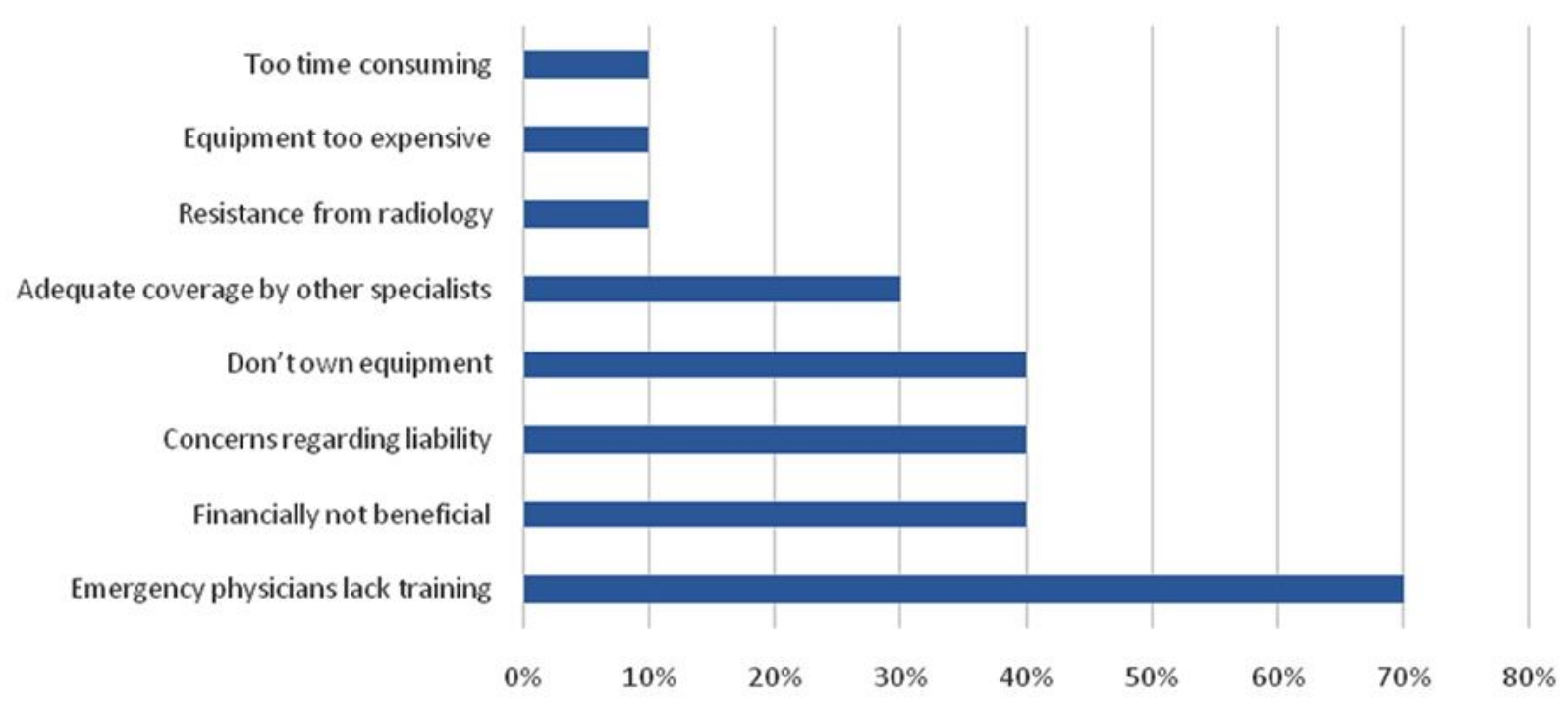

Figure 2. Response from ED directors about reasons for not implementing POCUS program.

ultrasound is equal or better than chest radiography for identifying a hemothorax or pneumothorax in trauma patients. Therefore, some trauma centers have begun performing an extended FAST exam (eFAST) for evaluating for pneumo- and hemothorax in addition to intraperitoneal injuries.

According to a report, only $54 \%$ of the more experienced ED physicians were found to be using POCUS in spite of 24-hour ultrasound accessibility and the newly trained EMs most of the time did not use POCUS (Bashir et al., 2016). Even though, a vast number of studies mentioned about numerous benefits of POCUS implementation, at present it has become essential to identify the barriers to POCUS utilization.

The present study illustrated the current practice of EPperformed POCUS in UAE EDs. The study detected that the use of POCUS in UAE government EDs is limited. A little more than half of UAE government EDs has been reported to own US machine. Quarter of EDs performed POCUS regularly, and less than half reported about quality assurance programs. Not only does this study identify several barriers to using POCUS routinely in UAE government EDs but it is a first of its kind study to fill in gaps unseen in the previous researches. The use of FAST in a particular institution or setting is based on access to equipment, appropriately trained personnel, and organized quality assurance program.

Lack of training was reported by $70 \%$ of ED directors to be the prime reason for POCUS being unused. Thus, there is a possibility that the $70 \%$ of the respondents, not implementing POCUS, would begin using POCUS if they had more training. Proper training of EMs in POCUS technique is essential for widespread implementation of POCUS. In order to enhance the number of new EPs educated in POCUS, academic centers would need to train residents in ultrasound and would need to recruit more ultrasound fellows to grow the subspecialty. However, there is a lack of uniformity in training programs related to POCUS skill development in undergraduate and graduate medical education (Kameda and Taniguchi, 2016). Additionally, EDs in each hospital and institution require distinctive skills and knowledge of ultrasound (Choi et al., 2017). Hence, some basic guidelines are required in developing nationwide educational programs for learning POCUS.

POCUS is actually a highly operator-dependent process, since its performance requires multiple skills, including) questioning about clinical findings and decision-making process, ii) requiring knowledge of sonographic windows in order to acquire optimized image quality, and iii) ability to interpret and integrate POCUS findings with other clinical data in order to take accurate clinical decision (Choi et al., 2017). If any one of the above-mentioned steps is missed, the true value of POCUS might be weakened. Thus, EPs trained on POCUS must be allowed to review the ultrasound images obtained from diversified cases. Hence, it is essential to save the ultrasound images reliably for quality control, as well as for educational and medico-legal purposes (Choi et al., 2017). Additionally, asynchronous learning platforms also made it easier to learn POCUS at little cost anytime from anywhere in the world. Education oriented websites, such as ACEP sonoguide.com provides free open-access medical education forums on websites, blogs, video log, and other Internet-based resources and enables real time education during actual scanning. Although this type of asynchronous training opportunity is increasing day- by day, the in-person training by faculty guided program is considered to be more effective in acquisition of POCUS skills by EPs (Sanders et al., 2015). Due to the rapid emergence of POCUS as a valuable tool for diagnosis of serious and life threatening 
conditions in EDs in both academic and private settings, ACEP has summarized the scope of practice of emergency ultrasound, its training pathways, documentation of US equipment, and quality assurance process (Adhikari et al., 2014).

Several respondents in this study reported that they could not use POCUS because of the unavailability and unaffordability of the US machine, equipment too expensive, and a lack of financially benefit the technique was not beneficial, indicating the need for more low-cost machines. Therefore, for increased POCUS usage, there is a requisite for targeted, low-cost POCUS devices producing good image quality.

Only $8.3 \%$ of EDs mentioned that they could bill separately for their emergency POCUS examination and majority of the respondents could not bill separately for POCUS studies. However, for effective POCUS use, financial integration of ultrasound into existing departmental billing and coding is essential. In fact, ACEP had developed and updated a comprehensive document about emergency ultrasound coding and reimbursement. The initiation of ultrasound billing and reimbursement program is critical to generate revenues that would cover the relevant costs associated with POCUS such as training for EPs, ultrasound equipment costs, image archival system, quality assurance of US machine, and also to provide incentives to EPs performing POCUS (Adhikari et al., 2014). Moreover, it is important to have uniformity in POCUS billing in EDs both locally and nationally.

About $40 \%$ of the respondents considered concerns for liability as the barrier for not performing POCUS in EDs. While concerns about liability with use of POCUS in the EDs persist, Blaivis and Pawl (2012) in a study detected that over a period of twenty years only one lawsuit was filed against an emergency physician due to failure to perform bedside ultrasound (Blaivas and Pawl, 2012; Bellows et al., 2015).

One of the limitations of the current study is that the survey might be limited by a recall bias due to selfreporting. This recall bias might affect the results. Nonetheless, it has been found that response to POCUS questions in the overall sample is more or less constant for most ED characteristics. However, it seems likely, that the data might be more accurate if measured at the level of individual EPs than at the level of ED directors. Another limitation could be the low response rate from the participants.

\section{CONCLUSION}

In conclusion, the present study identifies several barriers to using POCUS routinely in UAE government EDs. POCUS is mainly dependent on the skills of the operator and availability of physicians support for image interpretation. Lack of proper training has been detected to be the most common barrier to POCUS use in UAE
EDs.

The following suggestions might help to improve the use of POCUS in EDs: i) Encouraging ED administration to adapt POCUS by highlighting its role in patient care, patient safety, as well as potential source of revenue generation, ii) Facilitating improved faculty training by implementing a standardized credentialing process for various POCUS applications among all EDs. Faculty credentialing would increase POCUS use, even when the hospitals restrict POCUS because of limited privileges to perform ultrasound, and last but not the least iii) EDs should implement quality assurance program as described by international POCUS guidelines to ensure safe use of the skill, which will address the departments' concerns regarding legal liability. The quality assurance review could be enhanced by the use of web-based image archival system for improved documentation.

Future studies should be directed to define barriers to POCUS, since they might change with time and also to detect unique solutions to enhance POCUS utilization in the government EDs in the UAE.

\section{Conflict of interests}

The authors declare that there is no conflict of interest regarding publication of this article.

\section{REFERENCES}

Adhikari S, Amini R, Stolz L, O'Brian K, Gross A, Jones T, Fiorella A, Kleim SM, 2014. Implementation of a novel point-of -care ultrasound billing and reimbursement program: fiscal impact. Am J Emerg Med, 32: 592-595.

Atkinson P, Bowra J, Lambert M, Lamprecht H, Noble V, Jarman B, 2015. International Federation for Emergency Medicine point of care ultrasound curriculum. CJEM, 17: 161-170.

Bashir K, Chaudhury S, Bashir I, Cameron P, 2016. Physician's perspective on point-of-care ultrasound: Experience at a tertiary care emergency department in Qatar. J Emerg Med Trauma Acute Care, 9.

Bellows B, Totten J, Shah S, Adedipe A, 2015. Perceived barriers in the use of point of care ultrasound in the WWAMI region. J Emerg Med Crit Care, 1: 4.

Blaivas M, Pawl R, 2012. Analysis of lawsuit filed against emergency physicians for point-of-care emergency ultrasound examination performance and interpretation over a 20-year period. Am J Emerg Med, 30: 338-341.

Chen KC, Lin ACM, Chong CF, Wang TL, 2016. An overview of pointof-care ultrasound for soft tissue and musculoskeletal applications in the emergency department. J Intensive Care, 4: 55.

Choi YJ, Jung JY, Kwon H, 2017. Effectiveness of education in point-ofcare ultrasound-assisted physical examinations in an emergency department. Medicine, 96: e7269.

Fares S, Irfan FB, Corder RF, Al Marzouqi MA, AIZaabi AH, Idrees MM, Abbo M, 2014. Emergency medicine in the United Arab Emirates. Int J Emerg Med, 7: 4.

Herbst MK, Camargo CA Jr, Perez A, Moore CL, 2015. Use of point-ofcare ultrasound in Connecticut emergency departments. J Emerg Med, 48: 191-196.

Kameda T, Taniguchi N, 2016. Overview of point-of-care abdominal ultrasound in emergency and critical care. J Intensive Care, 4:53.

Moore CL, Molina AA, Lin $\mathrm{H}, 2006$. Ultrasonography in community emergency departments in the United States: Access to 
ultrasonography performed by consultants and status of emergency physician-performed ultrasonography. Ann Emerg Med, 47: 147-153.

Sanders JL, Noble VE, Raja AS, Sullivan AF, Carnargo CA, 2015. Access to and use of point-of-care ultrasound in the emergency department. West J Emerg Med, 16: 747-752.

Whitson MR, Mayo PH, 2016. Ultrasonography in the emergency department. Crit Care, 20: 227-234.
Citation: Arjandas V, Al Ameri FS, Chaudhry M, Buhumaid R, 2018.

The use of point of care ultrasound (POCUS) in UAE emergency departments (ED). Int Res J Med Med Sci, 6(1): 1-9. 


\section{Appendix}

\section{The use of Point-of-Care Ultrasound amongst Emergency Providers in the UAE}

1. Where is your hospital located?

a. Abu Dhabi

b. Abu Dhabi (Western region)

c. Dubai

d. Sharjah

e. Ajman

f. Umm Al-Quwain

g. Ras Al-Khaimah

h. Fujairah

2. How many patients does your emergency department see per year?

a. Less than 10,000

b. $\quad 10,000-20,000$

c. $\quad 21,000-40,000$

d. $\quad 41,000-60,000$

e. More than 60,000

3. How many full time physician's makeup your emergency department staff?

a. Less than 5

b. $\quad 5-15$

c. $\quad 15-30$

d. $\quad 30-45$

e. $\quad$ More than 45

4. What percentage of your emergency department staff is emergency medicine residency trained?

a. $\quad 1-25 \%$

b. $\quad 26-50 \%$

c. $\quad 51-75 \%$

d. $\quad$ More than $75 \%$

e. None

5. Of the emergency medicine-trained staff, what percentage graduated their emergency medicine residency in the last 10 years?
a. $\quad 1-25 \%$
b. $26-50 \%$
c. $\quad 51-75 \%$
d. More than $75 \%$
e. None
f. Not applicable

6. On average; how many ultrasound studies are ordered by your emergency department per week?

a. Less than 10

b. $\quad 10-20$

c. $\quad 21-40$

d. $\quad$ More than 40

7. Do you have limits on ordering ultrasound studies through radiology department?

a. $\quad$ Yes, we are requested to limit ultrasound orders

b. No, we have no limits on ordering ultrasound studies 
8. What is the typical time required to obtain the report of an ultrasound study from radiology during routine working hours (8am to $5 \mathrm{pm})$ ?
a. Less than 30 minutes
b. $\quad 30-59$ minutes
c. $\quad 60-89$ minutes
d. $\quad 90-120$ minutes
e. $\quad$ More than 2 hours
f. Not available

9. What is the typical time required to obtain the report of an ultrasound study from radiology after routine working hours (5pm to $8 \mathrm{am})$
a. $\quad$ Less than 30 minutes
b. $\quad 30-59$ minutes
c. $\quad 60-89$ minutes
d. $\quad 90-120$ minutes
e. $\quad$ More than 2 hours
f. Not available

10. Do physicians in your department perform Point-of-Care(bedside) ultrasound?

a. Regularly for many application

b. $\quad$ Regularly for certain applications only (eg. FAST, procedure guidance)

c. Occasionally

d. Never

11. Overall, how often emergency physicians in your department perform Point-of-Care ultrasound?

\begin{tabular}{|l|l|l|l|l|}
\hline & Regularly & Sometimes & Rarely & Never \\
\hline FAST & & & & \\
\hline Procedural & & & & \\
\hline Aorta & & & & \\
\hline Focused echo & & & & \\
\hline Biliary & & & & \\
\hline Renal & & & & \\
\hline Pelvic & & & & \\
\hline DVT & & & & \\
\hline Soft Tissue/ MSK & & & \\
\hline Other (specify) & & & \\
\hline
\end{tabular}

12. Do you have ultrasound machines in the emergency department?

a. An ultrasound machine is owned by the emergency department and available for use at all times (skip to question )

b. An ultrasound machine is barrowed from other departments (radiology, cardiology) for use by emergency physician if needed (skip to question )

c. We have no access to an ultrasound machine

13. Do you have plans to obtain access to emergency physician performed ultrasound?

a. Yes

b. No 
14. Which of the following are reasons for not implementing Point-of-Care ultrasound program in your emergency department (please check all that apply)
a. Emergency physicians lack training
b. Resistance from radiology
c. Adequate coverage by other specialists
d. Concerns regarding liability
e. Financially not beneficial
f. Too time consuming
g. Don't own equipment
h. Equipment too expensive
i. Other (please specify)

15. What is the minimal training level required by your hospital for physicians toperform Point-of-Care ultrasound?
a. $\quad$ Training course
b. Residency training
c. Fellowship Training
d. Peer training
e. $\quad$ others (please specify)
f. Not sure

16. Does your hospital have Point-of-Care ultrasound privileges?
a. Yes, global
b. Yes, applications specific
c. No
d. Other (please specify)

17. Do you have a formal Point-of-Care Ultrasound Quality assurance process?
a. Yes
b. No

18. Do you bill for ultrasound performed by Emergency physicians?

a. Yes

b. No

Thank you for your participation. 